\title{
Detection of clinically occult parotid gland and lymph nodal metastases in a case of meibomian gland carcinoma using 18-FDG PET CT
}

\author{
Prathamesh Vijay Joshi, Vikram Ramchandra Lele, Rozil Gandhi \\ Department of Nuclear Medicine and PET-CT, Jaslok Hospital and Research Centre, \\ Mumbai, India
}

\begin{abstract}
Meibomian gland carcinoma is a rare malignancy of the eyelid. We report a case of using 18-Flurodeoxyglocose Positron Emission Tomography-Computed Tomography (18-FDG PET-CT) for the detection of metastatic disease in a 60-year-old patient with meibomian gland carcinoma.
\end{abstract}

Key words: 18-FDG PET-CT, meibomian gland carcinoma, parotid gland, eyelid malignancy, metastasis, ocular neoplasm

\section{Case Report}

A 60-year old gentleman, a known case of meibomian gland carcinoma, was referred to our department for a whole body 18-F-FDG PET-CT scan. Six months before, he underwent excision of left upper eyelid swelling, clinically diagnosed as chalazion. The histopathology report revealed it to be a meibomian gland carcinoma. After three months he had local recurrence and left orbital exenteration was performed. Considering the aggressive course of the malignancy, a 18-F-FDG PET-CT study was performed to assess the whole body disease status. The scan revealed increased metabolic activity in the enlarged left parotid gland (Fig. 1) and left cervical lymph node (Fig. 2). No evidence of increased metabolic activity was noted at the surgical site (Fig. 3) and the rest of the body. We reported the left parotid gland and left supraclavicular lymph node as

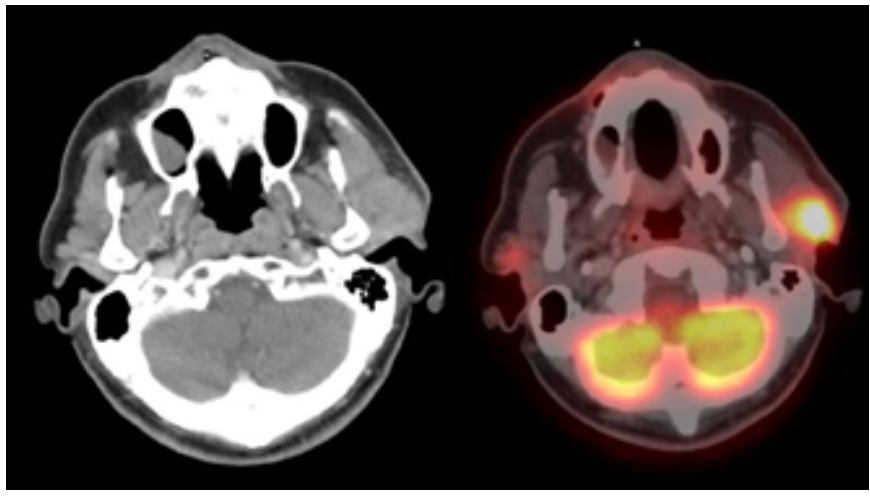

Fig. 1. CT and fusion PET-CT image showing metabolically active disease in heterogeneously enhancing enlarged left parotid gland. Standardized uptake maximum value (SUVmax) is $3.5 \mathrm{~cm} 2 / \mathrm{ml}$.

Correspondence: Dr Prathamesh Vijay Joshi, Department of Nuclear Medicine and PET-CT, Jaslok Hospital and Research Centre, Worli, Mumbai- 400026, India E-mail:drprathamj@gmail.com 


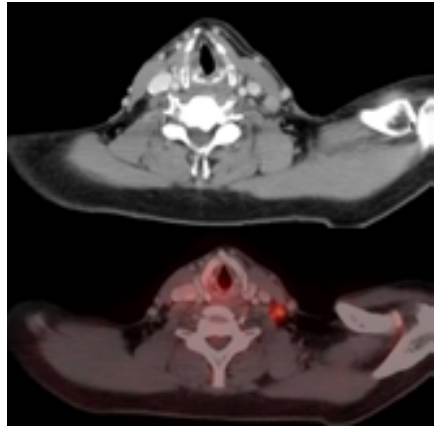

Fig. 2. CT and fusion PET-CT image showing metabolically active disease in left level IV lymph node. Standardized uptake maximum value (SUVmax) is $2.5 \mathrm{~cm} 2 / \mathrm{ml}$.

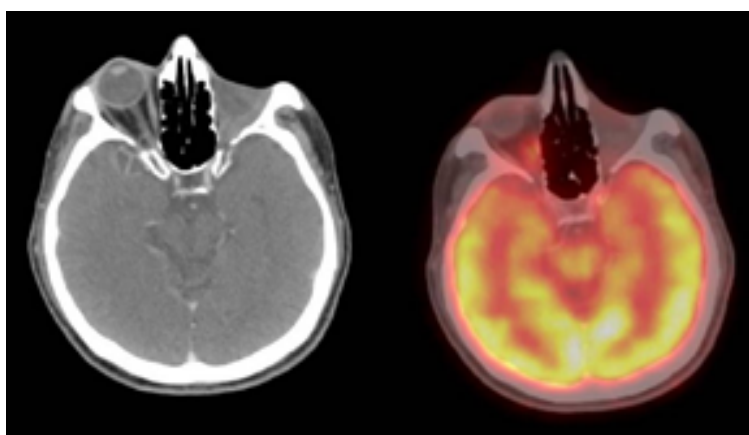

Fig. 3. CT and fusion PET-CT image showing no evidence of abnormal metabolic activity in left orbit (post surgery site). The FNAC of this region revealed no evidence of residual/ recurrent disease.

metastatic lesions. Histopathological examination of the left parotid gland and left supraclavicular gland after surgical excision confirmed metastatic meibomian gland carcinoma. Fine-needle aspiration cytology (FNAC) of the surgical site (where 18-F-FDG PET-CT did not show abnormal metabolic activity) did not reveal any residual disease.

\section{Discussion}

Cancer cells are known to show increased rates of glycolysis metabolism, ${ }^{1}$ and for this reason a PET study using [18F] fluorodeoxyglucose, a glucose analogue, has been used for the detection of primary and metastatic tumors. ${ }^{2}$ PET/CT, often regarded as the 'one-stop shop' for many malignancies, provides co-registered structural and metabolic images, allowing for accurate localization of sites of disease. This powerful imaging technique has minimized the suffering of a growing number of patients with serious diseases, including cancer, infection and inflammation, brain and cardiovascular disorders. ${ }^{3}$ In our case, we report the use of this extremely sensitive technique for diagnosing metastatic disease in a patient with meibomian gland carcinoma.

Meibomian gland carcinomas of the eyelid are rare neoplasms, accounting for less than $1 \%$ of all eyelid tumors. They usually mimic chalazia and undergo repeated curettage before a definitive diagnosis is made. ${ }^{4}$ Its an aggressive ocular neoplasm with potential for regional and distant metastasis. Early diagnosis and prompt surgical treatment can reduce mortality. ${ }^{5}$ This tumor occurs twice as often on the upper as on the lower eyelid, which reflects predominance of meibomian glands in the upper eyelid. ${ }^{6}$

Secondary spread to the parotid gland is usually through the lymphatic route. Many lymph nodes are present in the pre-auricular area, on the surface and within the parotid gland, and drain superficial tissues from the scalp (including upper eyelid, as in our case) and face. ${ }^{7}$

Use of FDG PET in staging this aggressive malignancy has been reported in the past in a case report. ${ }^{8}$ 


\section{Conclusion}

To conclude, our case suggests 18-F-FDG PET/CT can be used in restaging meibomian gland carcinoma. By diagnosing clinically occult metastases, FDG PET/CT can contribute in better management of this aggressive malignancy.

\section{References}

1. Weber G. Enzymology of cancer cell. N Eng J Med 1977;296:541-55I.

2. Hoh CK, Schiepers C, Seltzer MA, et al. PET in oncology will it replace the other modalities? Semin Nuci Med 1997;27:94-106.

3. Basu S, Alavi A, Unparalleled Contribution of 18F-FDG PET to Medicine Over 3 Decades J Nucl Med 49: $17 \mathrm{~N}-37 \mathrm{~N}$.

4. Mashburn, MA, Chonkich GD, Chase DR. Meibomian gland adenocarcinoma of the eyelid with preauricular lymph node metastasis. Laryngoscope1985;95:1441-1443.

5. Yasumura S, Shojaku H, Wada R, et al. Usefulness of Positron Emission Tomography for Management of Ocular Sebaceous Carcinoma. Clin Nuc Med 2010;35(2):98-100

6. Dailey RA, Ng JD. Sebaceous gland carcinoma. In: Roy FH, Fraunfelder F (eds.), Roy and Fraunfelder's Current Ocular Therapy, 6th ed. 2008; 272.

7. Seenu V, Misra MC, Khazanchi RK, et al. An eyelid swelling with a parotid mass. Postgrad Med J 1997;73(856):123-125.

8. Krishna SM, Finger PT, Chin K, et al. 18-FDG PET/CT staging of ocular sebaceous cell carcinoma. Graefe's Arch Clin Exp Opthalmol 245: 359-360. 